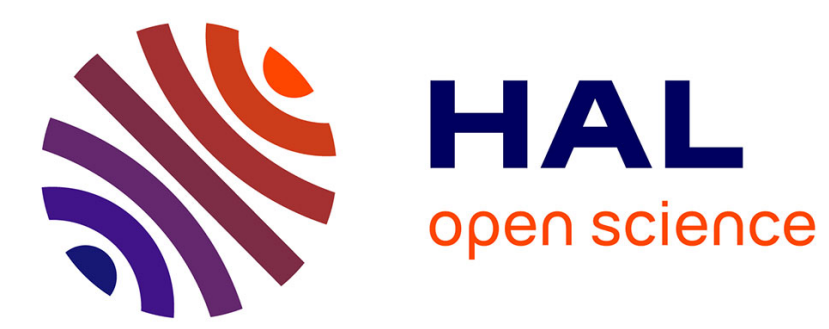

\title{
Diversité et fragilité des associations en Europe
}

Edith Archambault

\section{- To cite this version:}

Edith Archambault. Diversité et fragilité des associations en Europe. Informations sociales, 2012, 172, pp.20-28. halshs-00685130

\section{HAL Id: halshs-00685130 \\ https://shs.hal.science/halshs-00685130}

Submitted on 4 Apr 2012

HAL is a multi-disciplinary open access archive for the deposit and dissemination of scientific research documents, whether they are published or not. The documents may come from teaching and research institutions in France or abroad, or from public or private research centers.
L'archive ouverte pluridisciplinaire HAL, est destinée au dépôt et à la diffusion de documents scientifiques de niveau recherche, publiés ou non, émanant des établissements d'enseignement et de recherche français ou étrangers, des laboratoires publics ou privés. 


\section{Diversité et fragilité des associations en Europe}

\section{Édith Archambault}

Formant un modèle régional diversifié, les institutions sans but lucratif en Europe sont fragilisées par la crise économique et l'évolution libérale de la politique communautaire. Appauvries, transformées en sous-traitantes des programmes européens, leur innovation bridée, elles risquent, surtout dans le secteur social, de ne plus pouvoir comme aujourd'hui garantir une part importante du bien-être des Européens.

Si partout dans le monde existent des organismes à but non lucratif, leurs formes juridiques et leur degré d'institutionnalisation sont différents, ce qui rend les comparaisons internationales difficiles. Aussi les organisations internationales (ONU 2006, SNA 2008, chapitre 23) préconisent-elles de les répertorier non pas par leur forme juridique mais par un faisceau de cinq critères. Est alors considérée comme une institution sans but lucratif (ISBL) une structure :

- ayant un minimum d'organisation formelle, des réunions périodiques, par exemple, et une certaine durabilité ;

- sans but lucratif, c'est-à-dire ne distribuant pas de profits; l'organisation peut cependant accumuler des excédents qui seront affectés à sa mission fondamentale, à condition qu'aucune fraction n'en soit redistribuée aux membres, aux fondateurs ni aux administrateurs ;

- privée, c'est-à-dire institutionnellement distincte des administrations publiques ; I'organisation peut bénéficier d'un financement public notable ou même total et peut avoir des administrateurs représentants des pouvoirs publics, à condition qu'ils ne soient pas majoritaires ;

- auto-administrée, en mesure de conduire ses propres activités et sa gestion et de modifier de sa propre autorité ses statuts et son objet social ;

- à participation non obligatoire, ce qui signifie que la qualité de membre et les apports de temps et d'argent ne sont ni obligatoires, ni prescrits par la loi.

Les ISBL les plus répandues sont les associations et les fondations, dont les caractéristiques varient sensiblement selon le niveau de développement et I'environnement politique, social, religieux et culturel des pays. À la différence du modèle américain, le modèle européen est multiple. Pour rendre compte de la diversité des ISBL européennes, nous présenterons les cinq variantes identifiées dans les pays qui participèrent, au tournant du XXe siècle, au Programme Johns Hopkins de comparaison 
du secteur sans but lucratif (1), avant de montrer les mouvements de convergence entre ces variantes, puis l'ambiguïté des institutions européennes à l'égard des ISBL et, enfin, l'impact de la crise sur le tiers secteur européen (2).

\section{Le tiers secteur en Europe : un modèle, cinq variantes}

Les modèles européen et nord-américain sont contrastés à plus d'un titre. En Europe, les ISBL entretiennent partout une forme de partenariat avec les pouvoirs publics, plus ou moins ancienne, alors qu'aux États-Unis et dans une moindre mesure au Canada, elles privilégient l'initiative privée et entrent en compétition avec les services offerts par l'État et les entreprises lucratives. Ce rapport différent à l'État, joint au niveau plus bas de protection sociale en Amérique du Nord, explique que le financement public des ISBL prédomine en Europe, sous forme de subventions, de contrats ou d'achat de services,en se concentrant sur les celles qui œuvrent dans les domaines de l'État-providence, santé, éducation ou action sociale, alors qu'outre-Atlantique les ISBL comptent principalement sur leurs ressources propres : cotisations, participation des bénéficiaires au service rendu ou revenus de leur patrimoine. En Europe, la concurrence entre ISBL s'exerce plus au plan idéologique qu'économique; cette concurrence idéologique s'inscrit dans une histoire longue dont les origines remontent à l'Antiquité ou au Moyen-Âge, tandis qu'en Amérique du Nord prédomine un modèle puritain d'initiative privée charitable et de selfhelp qui remonte à un temps moins lointain, celui de la Conquête de l'Ouest.

Moins $15 \mathrm{mn}$.

En dépit de ces caractéristiques communes, toutefois, le modèle européen de tiers secteur n'est pas uniforme. Il varie d'un pays à l'autre selon les relations historiques et contemporaines des ISBL avec les pouvoirs publics à tous les niveaux, central, régional et local, et selon le caractère centralisé ou décentralisé de ces relations. Il varie aussi selon le niveau de protection sociale et le type du régime de sécurité sociale en vigueur bismarckien, beveridgien ou assistantiel (Esping-Andersen, 1990). Le niveau d'initiative et d'engagement bénévole de la population, son degré d'enracinement et son caractère complémentaire ou substituable à l'emploi salarié entraînent également des différences fondamentales entre pays européens, tandis que le niveau de l'emploi salarié est une bonne mesure du poids et de l'ancienneté du tiers secteur. Enfin, les religions principales, leur degré de concurrence et l'importance des liens historiques qu'elles entretiennent avec les ISBL façonnent également ces différences. L'existence de ces lignes de clivage a conduit à décliner le tiers secteur européen en cinq variantes: continentale, anglosaxonne, nordique, méditerranéenne ou orientale (3).

> La variante continentale (ou corporatiste): Allemagne, Autriche, Belgique, France, Pays-Bas, Suisse 
Les grandes ISBL de ces pays sont très professionnalisées dans les secteurs social, sanitaire et éducatif où elles sont fédérées plutôt idéologiquement selon la religion, le parti politique ou le syndicat. Le bénévolat, variable selon les pays, se rencontre principalement chez les petites organisations émergentes, innovantes et souvent plus militantes, dans les domaines de la culture, du sport et des loisirs ainsi que de la défense des droits ou des intérêts et de l'accompagnement des chômeurs vers l'emploi. C'est dans ces pays décentralisés que le financement public est le plus important, selon le principe de subsidiarité qui veut que l'État central n'intervienne pas directement quand une collectivité publique de plus petite taille ou une ISBL peut offrir un service de proximité. En contrepartie, les pouvoirs publics financent les ISBL qui offrent des services d'intérêt général ou collectif. Ce financement public provient plus des autorités locales (régions ou municipalités) et de la Sécurité sociale que de l'État central. Traditionnellement, les grandes ISBL ou leurs fédérations sont partenaires des pouvoirs publics dans l'expérimentation et la mise en œuvre des politiques publiques qui concernent leur champ d'activité.

> La variante anglo-saxonne (ou libérale): Irlande, Royaume-Uni

Les ISBL anglo-saxonnes ou voluntary organisations insistent sur la primauté symbolique du bénévolat, même si les plus importantes d'entre elles travaillent surtout avec des salariés. Le bénévolat, très répandu au Royaume-Uni et moins en Irlande, est une tradition plus ancienne que dans les pays continentaux : la Grande-Bretagne fut la première à instaurer en 1601 une loi sur les pauvres, qui est à l'origine de nombreuses charities. Cette valorisation du bénévolat obéit aussi à une logique puritaine qui veut que le travail soit une vocation et le bénévolat le meilleur usage du loisir, car il permet de rendre à la société ce que I'on a reçu d'elle. Aussi celui-ci se pratique-t-il dès le plus jeune âge à l'école, ainsi qu'à l'université où bénévoles et salariés des ISBL ont souvent suivi des cycles de formation spécialisés. Hôpitaux, musées, écoles et universités privées entrent en concurrence avec leurs homologues publics. Les ISBL de service social sont en relation étroite avec les collectivités locales qui procèdent à des appels d'offres où ces organisations volontaires entrent en compétition avec les entreprises lucratives. Les ressources du tiers secteur anglo-saxon sont plus variées et plus innovantes que dans le type continental, avec plus de ressources commerciales et davantage de dons directs ou canalisés par un réseau dense de fondations. Cependant, le financement public du secteur non lucratif reste prédominant dans ces deux pays européens, alors que les pays anglo-saxons nord-américains n'y ont que faiblement recours.

\section{$>$ La variante nordique (ou socio-démocrate) : Finlande, Norvège, Suède}

Le tiers secteur nordique est beaucoup moins important que dans les deux variantes précédentes : il ne représente que 2,3\% de l'emploi salarié contre 5,2\% en moyenne 
dans les pays continentaux et 6,5\% dans les pays anglo-saxons. Les ISBL nordiques sont de petite taille, peu professionnalisées et animées par de très nombreux bénévoles, principalement dans le secteur des sports et des loisirs et de la défense des consommateurs. Leurs ressources proviennent principalement de la participation des usagers et de dons. Le financement public est plus faible que dans les deux modèles précédents car l'État, avec le niveau de protection sociale le plus élevé du monde, fournit presque tous les services éducatifs, sanitaires et sociaux. Les inégalités sociales étant faibles, les ISBL humanitaires s'orientent vers les pays en développemen. Les fondations redistributrices sont très nombreuses, dont la plus symbolique est la Fondation Nobel en Suède.

\section{> La variante méditerranéenne (ou émergente) : Italie, Portugal, Espagne}

Le secteur non lucratif des pays méditerranéens est plus récent et moins développé que dans les deux premiers modèles (2,8\% de l'emploi salarié), car il a été restreint ou interdit pendant les périodes de dictature qu'ont connues ces pays au cours du XXe siècle. Il oppose un réseau d'ISBL catholiques, les plus anciennes, dans les secteurs de la santé, de l'éducation et de l'action sociale à un réseau laïque, souvent lié aux syndicats et aux coopératives. Le bénévolat organisé y est peu développé tandis que l'entraide informelle subsiste en milieu rural. Le niveau de protection sociale de ces pays est nettement moindre que dans les trois premières variantes. Les ressources des ISBL mêlent fonds public et ressources commerciales, avec un niveau de dons très faible. De fortes inégalités régionales sont à l'origine de nombreuses associations de développement local et le partenariat entre ISBL et régions apparaît depuis une dizaine d'années.

> La variante orientale (ou post-communiste): Hongrie, Pologne, République Tchèque, Roumanie, Slovaquie

C'est dans les pays d'Europe de l'Est que le secteur sans but lucratif est le moins important (0,8\% de l'emploi salarié), car la plupart des ISBL étaient interdites sous le régime communiste. Cependant, sa croissance est très rapide depuis la chute du Mur de Berlin, en partie sous l'impulsion de fondations américaines. Parallèlement la pratique du bénévolat se répand tout en restant très modeste. Avant 1989, seules les ISBL culturelles et de loisir étaient autorisées, l'État assurant santé, éducation et services sociaux comme dans le modèle scandinave, mais à un niveau évidemment moins élevé. Aussi les associations sportives culturelles et de loisir restent-elles les plus nombreuses, alors que la privatisation des services éducatifs sanitaires et sociaux a plus bénéficié au secteur lucratif qu'aux ISBL. C'est aussi dans les pays de l'Est que la dépense de protection sociale est la plus faible d'Europe. Les ressources commerciales y sont donc prépondérantes et les dons, fiscalement encouragés, relativement élevés. 
Ces modèles sont évidemment très schématiques et des pays hybrides assurent la transition entre ces différentes variantes du tiers secteur européen : entre les modèles continental et méditerranéen pour l'Italie et la France ; continental et nordique pour les Pays-Bas; anglo-saxon et continental pour l'Irlande; post-communiste et continental pour la Slovénie.

\section{Évolution récente des tiers secteurs européens et impact de la crise}

Au cours de la première décennie du XXIe siècle, on constate une certaine convergence des tiers secteurs européens vers un mélange des variantes continentale et anglosaxonne, accompagnée d'une professionnalisation croissante des ISBL, d'échanges entre organisations plus nombreux et plus internationaux et d'une diffusion des «bonnes pratiques » favorisée par les institutions européennes. Nous verrons d'abord quelles grandes tendances communes ont affecté les ISBL au cours de cette décennie, puis le rôle ambigu joué par les institutions européennes avant d'étudier l'impact de la crise sur les ISBL.

\section{> Les évolutions structurelles qui affectent le tiers secteur européen}

Au cours des dernières décennies, les grands pays européens centralisés ont connu une évolution vers un système politique et administratif plus décentralisé ; c'est évidemment le cas de la France avec les lois de décentralisation de 1983 et 2003 et du Royaume-Uni avec la Dévolution de 1999 qui a donné leur autonomie à l'Écosse et au Pays de Galles. Or la décentralisation favorise les réponses de proximité aux problèmes locaux, et donc les ISBL, lesquelles se sont multipliées au cours de la période récente partout en Europe. La tertiarisation de l'économie jointe à l'augmentation de l'emploi salarié féminin a également joué en faveur des ISBL, qui produisent principalement des services et emploient partout plus de femmes que d'hommes. Par ailleurs, cette montée du salariat féminin crée elle-même de nouveaux besoins en matière de garde d'enfants et d'accueil des personnes âgées dépendantes auxquels les structures publiques peuvent de moins en moins répondre; ce sont donc des opportunités supplémentaires pour les ISBL.

Des tendances démographiques et économiques lourdes sont aujourd'hui partagées par la plupart des pays européens avec une intensité variable : vieillissement de la population, faible niveau de fécondité, arrivée de populations immigrées de plus en plus lointaines, croissance insuffisante pour résorber un chômage de longue durée, paupérisation des bassins industriels traditionnels et désertification des zones rurales, montée des inégalités sociales. Ces changements posent des problèmes sociaux multiples, très divers localement, auxquels les ISBL sont aptes à répondre. Elles attirent partout, y compris dans les nouveaux pays membres de I'Union européenne, une classe moyenne de plus en plus éduquée et de plus en plus désireuse de prendre des initiatives pour apporter des solutions locales aux problèmes locaux. 
$>$ Vers une convergence des variantes européennes?

Face à ces évolutions structurelles communes, on observe des tendances à la convergence entre les variantes européennes de tiers secteur. Ces tendances sont renforcées par des échanges plus nombreux entre ISBL européennes et par les migrations ou le tourisme intra-européens. Le jumelage des villes européennes, par exemple, donne lieu la plupart du temps à des visites réciproques auxquelles participent les associations les plus représentatives de chaque ville en accompagnant maires et conseillers municipaux. Les coopérations entre ISBL des pays contigus se sont multipliées depuis l'entrée en vigueur de l'euro, qui permet des comparaisons de coût et de salaire immédiates ; on a vu apparaître les délocalisations de certains établissements médicosociaux dans les pays à moindre coût salarial ainsi que des déplacements de clientèle. Enfin, jusqu'à l'entrée en crise en 2008, les nouveaux pays membres de I'Union européenne ont connu des taux de croissance plus élevés que les anciens, ce qui leur a permis d'augmenter leur niveau de protection sociale, avec des systèmes mélangeant les modèles beveridgiens, bismarckiens et assistantiels, alors que les anciens pays membres tentaient de réduire le poids de leur protection sociale et prônaient un nouveau welfare mix où les ISBL prennent une part croissante et l'État une part décroissante.

Quelles sont les conséquences de ces tendances sur les cinq variantes du secteur non lucratif européen? Tout d'abord les variantes les plus récentes, méditerranéenne et orientale, s'estompent progressivement pour adopter un mélange continental/anglosaxon.

En second lieu, les variantes continentale et anglo-saxonne se rapprochent. La première devient moins corporatiste et plus commerciale : le principe de subsidiarité s'applique désormais aux entreprises et les collectivités publiques multiplient les appels d'offre. Simultanément la «pillarisation » c'est à dire le regroupement idéologique des ISBL, se réduit dans tous les pays continentaux, avec la sécularisation de la société et la moindre influence des partis politiques et des syndicats. Inversement, les ISBL anglo-saxonnes sont de plus en plus impliquées dans un partenariat avec l'État, par la signature d'engagements réciproques (compacts) pour combattre l'exclusion sociale et la pauvreté, notamment celle des enfants.

Enfin, la variante scandinave perd de son originalité : la réduction du niveau très élevé de dépense publique sociale a pour conséquence une réduction des services sociaux publics; des associations ou des coopératives sociales se sont créées dans la dernière décennie pour pallier ce retrait de l'État, mais aussi pour intégrer les vagues d'immigration importantes de la dernière décennie. Ces ISBL récentes sont beaucoup plus professionnalisées que les anciennes.

Ainsi s'esquisse un mouvement de convergence des cinq variantes que les institutions européennes ont plus ou moins accompagné. 


\section{> La position ambiguë des institutions européennes}

La Commission européenne et le Parlement européen affirment périodiquement que les ISBL sont essentielles au maintien de la cohésion sociale et constituent une part de I'identité européenne. L'Année européenne du bénévolat et du volontariat a été l'occasion, en 2011, de célébrer l'engagement citoyen sur lequel repose la plupart des ISBL. Par ailleurs la Commission et le Parlement encouragent la création de regroupements européens des ISBL qui travaillent dans les mêmes domaines, dont ils sollicitent l'avis sur les textes qui les concernent et reçoivent les lobbyistes. Enfin le Conseil économique et social européen récemment créé représente, comme en France, la société civile organisée et inclut un groupe des associations.

En dépit de ces signes de reconnaissance de l'utilité sociale et de la spécificité des ISBL, les institutions européennes soumettent celles-ci aux mêmes règles de concurrence que les entreprises lucratives et considèrent que les pouvoirs publics, en les subventionnant, faussent cette concurrence, notamment dans le domaine très important des services sociaux. Ce faisant, la politique européenne pousse les États à recourir à la commande publique et aux appels d'offres et banalisent ainsi les ISBL qui perdent leur capacité d'initiative. La lente négociation sur les services sociaux d'intérêt général témoigne de la lutte contre cette banalisation. Enfin, le statut d'association européenne n'a toujours pas été voté alors qu'un texte existe depuis plus de vingt ans et que ce statut permettrait aux grandes associations implantées dans plusieurs pays de coopérer plus facilement.

\section{> L'impact de la crise}

La crise financière et bancaire de 2008 et celle des dettes souveraines que la zone euro traverse actuellement ont des répercussions économiques et sociales dans tous les pays européens. Les ISBL y sont soumises à un effet de ciseau, avec une augmentation du nombre de personnes défavorisées qui s'adressent à elles alors que leur financement public baisse sensiblement. Si cette conjoncture affecte plus particulièrement les ISBL de services sociaux, très importantes dans la variante continentale, toutes sont frappées.

Face à cette double crise, les ISBL sont amenées à être plus sélectives dans leur programmes, à réduire leur emploi salarié et à rechercher d'autres sources de financement privé ; elles se tournent notamment du côté des entreprises, car la progression des dons ne peut être que limitée, les Européens n'ayant, en 2011, toujours pas retrouvé leur niveau de revenu de 2008, sauf dans quelques pays. Elles auront également tendance à augmenter la participation financière des usagers au service qui leur est rendu, ce qui élimine les publics les moins solvables. Des mouvements de concentration et de mutualisation de moyens humains ou financiers s'observent sous la pression de la nécessité. Ces restructurations seront durables; elles sont pour une part rationnelles, mais elles réduisent aussi la proximité des ISBL avec leurs bénéficiaires. 
Enfin, la tendance des États à sous-traiter une part des services publics aux moinsdisants, via des appels d'offres de plus en plus précis, transforme les ISBL en soustraitants et réduit leur capacité d'initiative et d'innovation. La logique gestionnaire qui prévaut alors dans ces ISBL devenues des instruments des pouvoirs publics décourage le travail bénévole; les plus militants se retrouvent dans les organisations les plus récentes, plus engagées et plus proches des situations locales.

Le pire n'est jamais sûr. Dans tous les pays européens, les ISBL font preuve d'intelligence pour fonctionner avec des moyens limités et de leur aptitude à innover, à récupérer, à mettre en mouvement les bonnes volontés. Elles n'en sont pas moins des structures fragiles qui disparaîtront silencieusement si les États impécunieux tendent à appliquer les ajustements financiers inévitables à leurs partenaires les moins bruyants. On sait pourtant qu'une part importante du bien-être des nations européennes repose sur les ISBL. Celles-ci offrent des services éducatifs, sanitaires et sociaux de qualité et de proximité, démocratisent la culture et le sport, incitent les citoyens à participer à la vie politique, créent ou recréent les liens sociaux et tissent une société civile qui ne saurait vivre que d'échanges marchands (Stiglitz, Sen et Fitoussi, 2009). Enfin, grâce à leurs échanges internationaux, elles contribuent à créer pas à pas l'Europe des peuples et à combler le déficit démocratique indéniable de I'Union européenne.

\section{Notes}

1 - À l'initiative des Professeurs Lester Salamon (Johns Hopkins University) et Helmut Anheier (London School of Economics), un programme de comparaison internationale du secteur sans but lucratif a été lancé en 1990. Il s'est déroulé en deux phases (1990-95, 13 pays et 1996-2002, 42 pays). La partie française de cette recherche, placée sous la direction d'Édith Archambault, a été financée principalement par la Fondation de France. 2 - Pour une présentation plus approfondie et plus étayée du présent article, voir Archambault, 2001 et 2009.

3 - Les pays cités sont ceux qui ont participé au Programme Johns Hopkins de comparaison internationale du secteur sans but lucratif au cours de la phase 2 . Ces pays comptent environ $90 \%$ de la population de I'Union européenne (Salamon, Sokolowski et alii, 2004).

\section{Bibliographie}

Archambault E., 2001, « $\mathbf{Y}$ a-t-il un modèle européen de secteur sans but lucratif ? », Recma (Revue internationale de l'économie sociale), Paris, n²82, p. 64-83.

Archambault E., 2009, «The Third Sector in Europe: Does it Exhibit a Converging 
Movement » in Enjolras B. et K. H. Sivesind E. (dir.), Civil Society in Comparative Perspective, Comparative Social Research, vol. 26, Emerald Group Publishing Ltd.

Esping-Andersen G., 1990, The Three Worlds of Welfare Capitalism, Cambridge, Polity Press et Princeton, Princeton University Press.

Salamon L. M., Sokolowski S. W. et alii, 2004, Global Civil Society; Dimensions of the Nonprofit Sector, vol. 2, Kumarian Press.

Stiglitz J., Sen A. et Fitoussi J.-P., 2009, Rapport de la Commission sur la mesure de la performance économique et du progrès social, La Documentation française.

Nations Unies, 2006, Manuel sur les institutions sans but lucratif dans le Système de comptabilité nationale, Etudes méthodologiques, série $\mathrm{F}, \mathrm{n} \times 91$

System of National Accounts 2008 (SNA), « Non-profit institutions », chapitre 23, United Nations Statistical Commission (UNSC),

http://unstats.un.org/unsd/nationalaccount/sna2008.asp 